\title{
Avaliação da limpeza de CDtrodo construídos a partir de CD de ouro gravável/fita adesiva de galvanoplastia
}

\author{
M. V. Foguel, C. V. Uliana, P. R. U. Tomaz, P. R. B. Oliveira Marques, \\ H. Yamanaka, A. A. Pupim Ferreira* \\ UNESP - Universidade Estadual Paulista, Instituto de Química, \\ Campus de Araraquara. CP 355, 14800-900 Araraquara, SP, Brasil. \\ Autor correspondente: antoferr@iq.unesp.br \\ Brazil
}

\begin{abstract}
Resumo: Os eletrodos de ouro são largamente utilizados em estudos eletroquímicos e eletroanalíticos, devido à sua elevada pureza, ampla faixa de potencial de trabalho, bem como a possibilidade de controle e modificação da superfície eletródica. Neste trabalho são descritos procedimentos para construção e limpeza de eletrodos a partir de CDs de ouro graváveis, denominados CDtrodos. Inicialmente, os CDs foram submetidos à ação de $\mathrm{HNO}_{3}$ concentrado para retirada da camada polimérica protetora e exposição da camada metálica, posteriormente, para a construção dos CDtrodos, delimitou-se a área eletródica com fita de galvanoplastia. Diversos métodos para a limpeza da superfície do ouro foram empregados, após ataque do $\mathrm{HNO}_{3}$, tais como, aplicação de potencial fixo em solução de $\mathrm{NaCl}$, ciclagens sucessivas em $\mathrm{H}_{2} \mathrm{SO}_{4}$ e aplicação de ultra-som, sendo que os melhores resultados foram obtidos por tratamento com $\mathrm{H}_{2} \mathrm{SO}_{4}$. A literatura tem registrado vários trabalhos empregando CDtrodo no entanto são focados na aplicação e pouco se descreve sobre a limpeza do eletrodo; nenhum trabalho foi registrado empregando a fita de galvanoplastia para delimitar a área do eletrodo. A utilização desse tipo alternativo de eletrodo é de suma importância, uma vez que o mesmo apresenta desempenho eletroquímico comparável aos eletrodos comerciais, além de grande versatilidade e baixo custo.
\end{abstract}

Palavras-Chaves: CDtrodo; eletrodo CD-R; voltametria cíclica; limpeza de superfície eletródica.

\section{INTRODUÇÃO}

O emprego de superfícies metálicas na construção de sensores eletroquímicos tem levado ao desenvolvimento dos métodos eletroanalíticos. Os eletrodos metálicos (Au, Pt, dentre outros) possibilitam efetuar trabalhos em um vasto intervalo de potencial permitindo ainda o controle e modificações em sua superfície de trabalho [1]. Os transdutores de ouro merecem destaque em aplicações em eletroquímica e eletroanalítica e, recentemente, métodos alternativos de obtenção de eletrodos têm sido propostos como no caso de eletrodos obtidos a partir de discos compactos graváveis (CD-Rs) [2-4]. CD-Rs de ouro são constituídos por uma base de policarbonato acrescida de uma camada fotossensível (cianina, ftalocianina ou azo compostos), na qual ocorre o processo de gravação. Sobre este filme é depositada, pelo processo de sputtering, uma fina camada de ouro com espessura variando entre 50 e $100 \mathrm{~nm}$ e área total da ordem de $100 \mathrm{~cm}^{2}$, que é recoberta por um ou dois filmes poliméricos protetores.

No comércio existem diferentes marcas de CD-Rs e que podem diferir quanto ao tipo de composto fotodegradável, camadas protetoras, com- 
posição e depósito do filme de metal utilizado em sua produção. Isto significa que os procedimentos empregados na remoção dos filmes poliméricos e pré-tratamento de superfície, para obtenção de um eletrodo com superior versatilidade, podem ser dependentes da procedência dos CD-Rs. Os eletrodos de ouro (CDtrodos) têm sido preparados conforme procedimento descrito por Angnes et al. [2]. São construídos de forma simples com baixo custo financeiro (a partir de um CD-R podem ser obtidos vários eletrodos) sendo, portanto uma alternativa promissora na obtenção de sensores eletroanalíticos reprodutíveis e com possibilidades de modificações de suas superfícies de trabalho. Os CDtrodos de ouro têm performance eletroquímica comparável aos eletrodos convencionais, com superior versatilidade. Uma possibilidade atraente é a da utilização na construção de eletrodos na forma descartável, que compensa os problemas apresentados pela superfície de ouro como os processos de envenenamento decorrentes da formação de óxidos de ouro.

Trabalhos na literatura relatam a modificação CDtrodos de ouro com monocamada autoorganizada (self-assembled monolayers) [5], emprego na determinação de diferentes analítos em várias matrizes, tais como, dipirona em formulações farmacêuticas [6], cobre em cana-de-açúcar e água de torneira [7] e em meio hidroalcóolico [8], chumbo em água de chuva [9], Ce(IV) [10] e Se (IV) [11] em água deionizada. Esses trabalhos utilizam materiais como esmalte, resina epóxi, PVC e tinta de impressora, para delimitação da área geométrica do eletrodo, o que necessita de grande precisão na construção dos mesmos, para obtenção de uma área superficial reprodutível com relação ao sinal elétrico medido.

O presente trabalho apresenta estudos sobre a construção de CDtrodos de ouro empregando fita adesiva para galvanoplastia a fim de delimitar a área de trabalho eletródica e investigação sobre os procedimentos de limpeza e pré-tratamento da superfícies do eletrodo de ouro construído a partir de CDs graváveis.

\section{MATERIAIS E MÉTODOS}

\section{Reagentes e soluções}

Solução tampão fosfato de sódio $0,1 \mathrm{~mol}$ $\mathrm{L}^{-1}, \mathrm{pH}$ 7,0 (eletrólito de suporte) foi preparada a partir dos sais $\mathrm{Na}_{2} \mathrm{HPO}_{4} / \mathrm{NaH}_{2} \mathrm{PO}_{4}$ (Merck), conforme descrito na literatura [12]. Nos experimentos de voltametria cíclica foram empregados eletrólito de suporte contendo a espécie redox $\mathrm{Fe}(\mathrm{CN})_{6}^{4-}$ (Sigma-Aldrich). Ácido sulfúrico $\left(\mathrm{H}_{2} \mathrm{SO}_{4}\right)$ e cloreto de sódio $(\mathrm{NaCl})$, utilizados na ativação da superfície do CDtrodo de ouro, foram fornecidos pela Mallinckrodt. Ácido nítrico $\left(\mathrm{HNO}_{3}\right.$ ) foi fornecido pela MERCK. A fita adesiva para galvonoplastia 470 e alumina de polimento metalográfico $(0,05 \mu)$ foram fornecidas, respectivamente, pela $3 \mathrm{M}$ e Arotec. Todas as soluções foram preparadas em água Milli-Q ( $\rho>18,2 \mathrm{M} \Omega \mathrm{cm}$, Millipore Inc., USA).

\section{Equipamentos, eletrodos e célula eletro- química}

As medidas eletroquímicas foram obtidas, a temperatura ambiente, com o auxílio de um potenciostato/galvanostato EG\&G PAR, modelo 263, conectado a um microcomputador com o software 4.30 (EG\&G) para aquisição e análise dos dados obtidos. Uma célula eletroquímica de compartimento único de $5 \mathrm{~mL}$ contendo o eletrólito de suporte e as espécies redox $\mathrm{Fe}(\mathrm{CN})_{6}{ }^{4-}$ foi utilizada para as medidas, juntamente com um sistema de três eletrodos: CDtrodo de ouro $\left(\mathrm{A}_{\text {geom }}=0,071 \mathrm{~cm}^{2}\right), \mathrm{Ag}|\mathrm{AgCl}| \mathrm{KCl}_{\text {(sat) }}$ e fio de platina $\left(A_{\text {geom }}=4 \mathrm{~cm}^{2}\right)$ como eletrodo de trabalho $\left(E_{T}\right)$, referência e auxiliar, respectivamente. Para a etapa em ultra-som, utilizou-se um equipamento de marca Iunic, modelo USC-700. Para obtenção de micrografias foi utilizado o equipamento microscópio de varredura eletrônica (scanning electron microscope SEM) JEOL-JSM-T330 A.

\section{Construção de eletrodos de ouro (CDtro- dos)}


A literatura registra a construção de CDtrodo empregando tinta de impressora [4], espaçador de teflon [10] e resina epóxi $[2,11]$ para delimitar a área geométrica do eletrodo de trabalho. No presente trabalho propõe-se a utilização de fita de galvanoplastia como alternativa para este fim, facilitando a construção do CDtrodo. Inicialmente, para a obtenção de eletrodos metálicos a partir de CD-R de ouro faz-se necessária retirar a camada de proteção constituída por filmes poliméricos para ter acesso à camada metálica. Foram utilizados discos compactos graváveis (marca Mitsui Archive Gold CD-R 100) e aplicado o seguinte procedimento na construção dos CDtrodos: a) retirada das camadas poliméricas protetoras com $\mathrm{HNO}_{3}$ concentrado; b) lavagem da superfície de ouro com água destilada; c) corte da superfície CD-R, nas dimensões em torno de $2 \mathrm{~cm}$ de comprimento por $0,5 \mathrm{~cm}$ de largura; d) definição da área do eletrodo de trabalho com fita adesiva de galvanoplastia, perfurada empregando furador de rolhas (diâmetro de 3,0 $\mathrm{mm}$ ) e fixada sobre o filme de ouro; e) contato elétrico do CDtrodo, fixando uma lâmina de cobre (fio de cobre laminado e polido com lixa 3M 800) com fita de PTFE isolante (politetrafluoretileno). A Figura 1 representa o esquema de construção do CDtrodo.

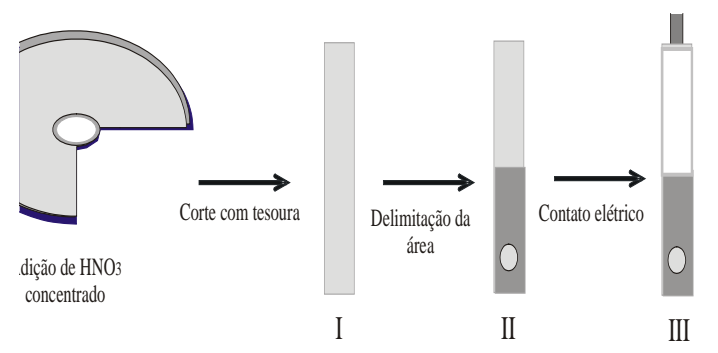

Figura 1. Construção de CDtrodos: I) área recortada do CD; II) delimitação da área do eletrodo empregando fita de adesiva de galvanoplastia; III) fixação de cobre laminado (contato elétrico) por meio da fita de teflon.

\section{Pré-tratamento da superfície de ouro}

Após o preparo do CDtrodo de ouro, fezse necessário uma limpeza da superfície para a remoção da camada de óxido formado pela ação do ácido nítrico concentrado sobre a camada metálica durante a remoção das camadas protetoras. Foram estudados os seguintes procedimentos para a ativação da superfície de ouro:

I) Aplicação de ultra-som em meio de água ou etanol durante 5 min;

II) Limpeza mecânica com alumina;

III) Aplicação de potencial fixo $+0,5 \mathrm{~V}$ durante 5 min em uma solução de $\mathrm{NaCl} 0,5 \mathrm{~mol} \mathrm{~L}^{-1}$;

IV) Ciclagens sucessivas em $\mathrm{H}_{2} \mathrm{SO}_{4} 0,5 \mathrm{~mol}$ $\mathrm{L}^{-1}$ no intervalo de potencial entre 0,0 e $+1,5 \mathrm{~V}$ e velocidade de varredura igual a $100 \mathrm{mV} \mathrm{s}^{-1}$;

\section{Avaliação do desempenho dos CDtrodos de ouro}

Após a etapa de pré-ativação da superfície, o CDtrodo-Au foi avaliado pela técnica de voltametria cíclica, por meio do comportamento da espécie redox, em termos de variação de potencial de pico e de corrente de pico, comparando-se os dados para cada etapa avaliada.

Os voltamogramas foram traçados em eletrólito de suporte contendo a espécie redox $\mathrm{Fe}(\mathrm{CN})_{6}^{4-} 1 \times 10^{-2} \mathrm{~mol} \mathrm{~L}^{-1}$, no intervalo de potencial de $-0,4 \mathrm{a}+0,7 \mathrm{~V}$ e com velocidade de varredura de $50 \mathrm{mV} \mathrm{s}^{-1}$.

Microscopia de varredura eletrônica foi empregada para comparar as superfícies do CDtrodos.

\section{RESULTADOS E DISCUSSÃO}

A retirada manual das camadas poliméricas do CD-R para então expor a camada de ouro apresentou voltamograma cíclico do sistema padrão (solução tampão fosfato $+\mathrm{Fe}(\mathrm{CN})_{6}{ }^{4-}$ ) conforme esperado, no entanto como a camada de ouro é muito delgada a não reprodutibilidade na confecção do CDtrodo indicou inviabilidade do procedimento. Dessa forma, o ácido nítrico foi em- 
pregado para remoção da camada polimérica nos estudos subseqüentes.

A literatura tem registrado o emprego imediato do CDtrodo, sem qualquer tratamento prévio $[4,5,11]$ ou que o CDtrodo recém preparado apresenta excelente resposta mas quando estocado deve ser ativado por meio de ciclagens em solução de $\mathrm{HClO}_{4}$ [9]. Assim, imediatamente após a construção do CDtrodo, no qual foi empregado ácido nítrico concentrado na retirada das camadas protetoras, foram traçados voltamogramas cíclicos em solução tampão fosfato de sódio 0,1 mol L-1, pH 7,0 e contendo $\mathrm{Fe}(\mathrm{CN})_{6}^{4-} 1,0 \times 10^{-2}$ $\mathrm{mol} \mathrm{L} \mathrm{L}^{-1}$, no intervalo de potencial de $-0,4 \mathrm{a}+0,7 \mathrm{~V}$, $v=50 \mathrm{mV} \mathrm{s}^{-1}$. Observa-se na Figura 2 que os picos que caracterizam o sistema eletroquímico padrão, encontram-se bastante deslocados (655 mV de diferença) e deformados, ocasionados, possivelmente, pela dificuldade da transferência eletrônica na superfície do eletrodo, como indicado pela razão entre os picos catódico e anódico. Isto pode ser atribuído à presença de óxidos de ouro formados pelo ataque ácido $\left(\mathrm{HNO}_{3}\right)$ na retirada das camadas protetoras e a provável presença de material residual das camadas poliméricas de proteção sobre a superfície de ouro.

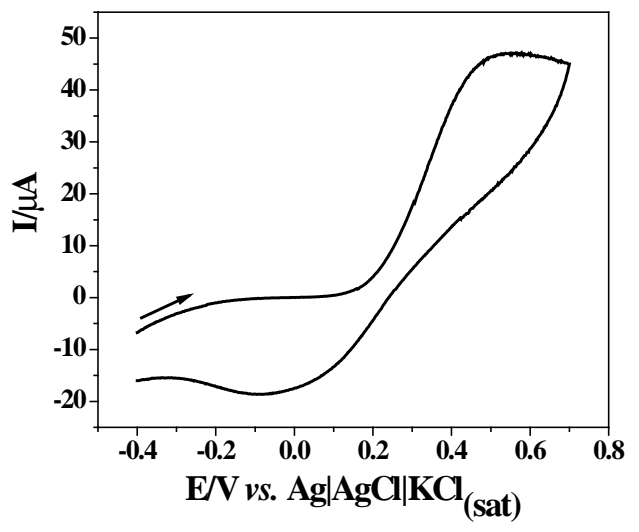

Figura 2. Voltamograma cíclico em solução tampão fosfato de sódio $0,1 \mathrm{~mol} \mathrm{~L}^{-1}$, $\mathrm{pH} \mathrm{7,0} \mathrm{contendo}$ $\mathrm{Fe}(\mathrm{CN})_{6}{ }^{4-} 1,0 \times 10^{-2} \mathrm{~mol} \mathrm{~L}^{-1}$ sobre CDtrodo de ouro, antes de qualquer tipo de limpeza da superfície eletródica; $v=50 \mathrm{mV} \mathrm{s}^{-1}$.
A Figura 2 demonstra a necessidade de limpeza da área eletródica, após a ação do $\mathrm{HNO}_{3}$. Foram investigadas limpezas por meio da aplicação de ultra-som sobre a superfície de ouro do CD, tanto em água como em etanol, antes das medidas eletroanalíticas. No entanto, esses procedimentos ocasionaram o desprendimento da fina camada de ouro da superfície do CDtrodo, sendo então considerados inapropriados.

Foi investigado um procedimento de limpeza mecânica empregando solução de alumina $(0,05 \mu)$. A Figura 3 indica que os picos relativos à oxidação e redução apresentaram-se melhor definidos, com $\Delta \mathrm{E}_{\text {pico }}$ em trono de $90 \mathrm{mV}$ e razão de corrente de pico igual à unidade.

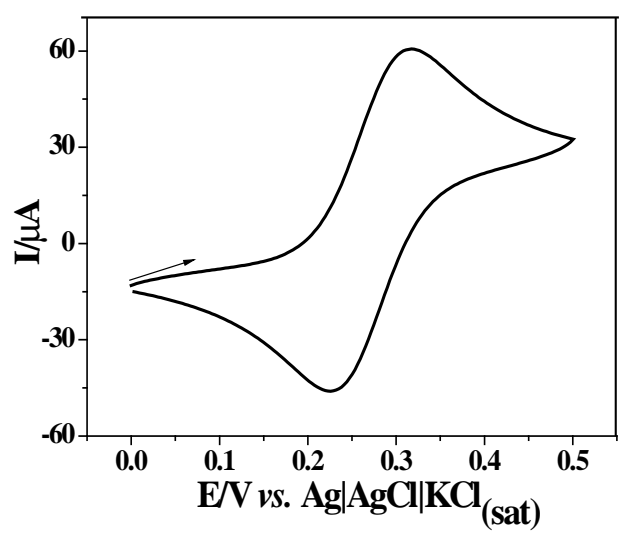

Figura 3. Voltametria cíclica em solução tampão fosfato $0,1 \mathrm{~mol} \mathrm{~L}^{-1}$, $\mathrm{pH} 7,0$ contendo $\mathrm{Fe}(\mathrm{CN})_{6}^{4-}$ $1 \times 10^{-2} \mathrm{~mol} \mathrm{~L}^{-1}$. E com alumina; $v=50 \mathrm{mV} \mathrm{s}^{-1}$.

Empregando-se outro CDtrodo foi efetuado um estudo do efeito da variação de velocidade de varredura cujos resultados estão representados na Figura 4. Pode se observar que o par redox foi deslocado com o aumento da velocidade de varredura no sistema, o que indica complicações cinéticas nos processos eletródicos. Isso se deve provavelmente à resíduos de óxidos na superfície de ouro não totalmente eliminado na limpeza manual; o emprego da alumina requer muito cuidado para que a camada de ouro não seja retirada 


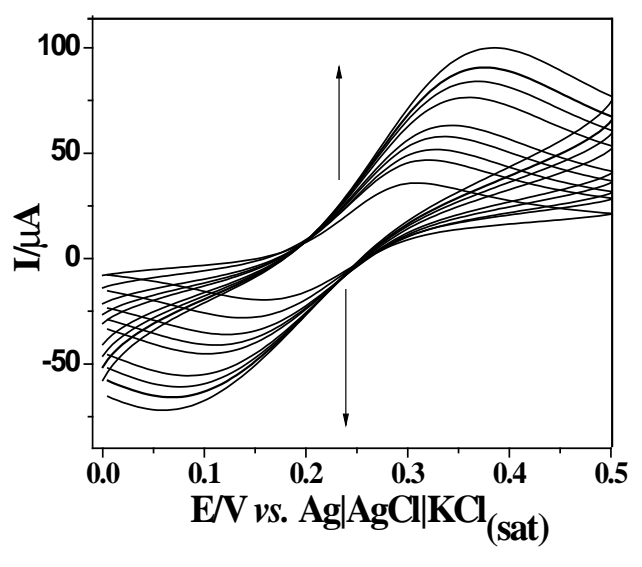

Figura 4. Efeito da velocidade de varredura nos voltamogramas cíclicos em solução tampão fosfato $0,1 \mathrm{~mol} \mathrm{~L}^{-1}$, pH 7,0 contendo Fe(CN) ${ }_{6}^{4-} 1$ x $10^{-2} \mathrm{~mol}$ $\mathrm{L}^{-1}$ empregando os seguintes valores: $10,20,30,40$, 50, 80, 100, 120, $150 \mathrm{mV} \mathrm{s}^{-1}$.

Investigação empregando microscopia de varredura eletrônica foi efetuada, sobre a superfície do CDtrodo limpo pelo procedimento mecânico com suspensão de alumina, objetivando avaliar se a mesma poderia alterar a superfície de ouro, visto que esta camada de ouro, depositada sobre o suporte de policarbonato, é muito fina. A Figura 5 apresenta os dados microscópicos obtidos nesta etapa de trabalho. Observa-se claramente que o uso da alumina acarretou danos na superfície do $\mathrm{CD}$, alterando assim, a sua área de trabalho, o que torna inviável a construção de eletrodos de CDs reprodutivos fazendo-se uso deste procedimento de limpeza.
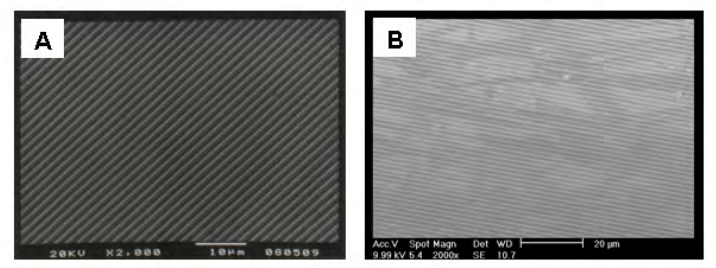

Figura 5. Micrografias de varredura eletrônica da superfície de CD de ouro. A) CD sem limpeza mecânica com alumina (2000x). B) CD após limpeza mecânica com alumina (2000x).
A literatura registra um tratamento efetuado com aplicação de potencial fixo em solução contendo cloreto, indicando que tal pré-tratamento foi o suficiente para limpeza da superfície de ouro [13]. Baseando-se neste procedimento, foi proposto efetuar uma limpeza eletroquímica, na qual o CDtrodo foi submetido à aplicação de potencial fixo em $+0,5 \mathrm{~V}$ por 5 minutos em solução de $\mathrm{NaCl}$ $0,5 \mathrm{~mol} \mathrm{~L}^{-1}$. A ativação da superfície foi então novamente avaliada pela técnica de voltametria cíclica, em meio de $\mathrm{Fe}(\mathrm{CN})_{6}{ }^{4-}$. Observou-se uma melhora no perfil voltamétrico do par redox, como pode ser notada na Figura 6. No entanto, os picos ainda apresentaram deslocamento de potencial de pico de aproximadamente $260 \mathrm{mV}$ e razão entre as correntes de pico catódica e anódica em torno de 0,61 , demonstrando a necessidade de uma limpeza mais eficiente.

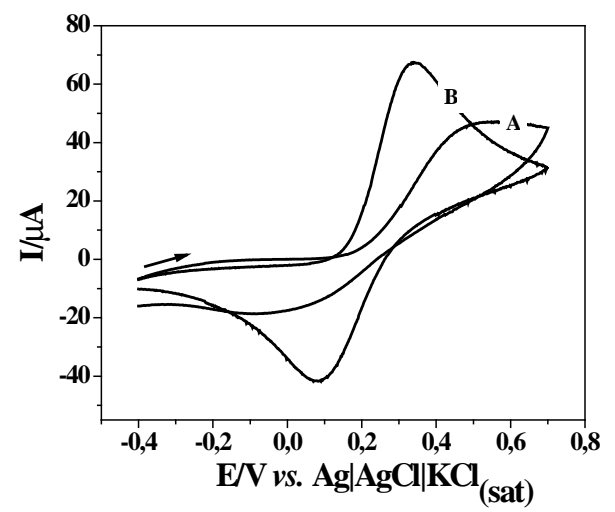

Figura 6. Voltamogramas cíclicos em solução tampão fosfato $0,1 \mathrm{~mol} \mathrm{~L}^{-1}$, $\mathrm{pH} 7,0$ contendo $\mathrm{Fe}(\mathrm{CN})_{6}^{4-}$ $1,0 \times 10^{-2} \mathrm{~mol} \mathrm{~L}^{-1}$ antes (A) e após (B) limpeza da superfície do CDtrodo de ouro aplicando potencial fixo $+0,5 \mathrm{~V}$ em solução de $\mathrm{NaCl} 0,5 \mathrm{~mol} \mathrm{~L}^{-1}$ durante $5 \mathrm{~min} ; \mathrm{v}=50 \mathrm{mV} \mathrm{s}^{-1}$.

Posteriormente, o procedimento de limpeza eletroquímica em meio de ácido sulfúrico foi investigado. Varreduras sucessivas foram traçadas em solução de $\mathrm{H}_{2} \mathrm{SO}_{4} 0,5 \mathrm{~mol} \mathrm{~L}^{-1}$ no intervalo de potencial de $0,0 \mathrm{~V}$ a $+1,5 \mathrm{~V}$, à velocidade de varredura de $100 \mathrm{mV} \mathrm{s}^{-1}$, até obtenção do voltamograma cíclico típico para o meio [14]. Nos primeiros ciclos, os voltamogramas apresentaram-se um pouco distintos, porém a partir de 30 ciclos 
o sinal apresentou-se bastante estável e similar a um eletrodo de ouro comercial $[2,15,16]$. Foram observados dois picos característicos, um pico anódico em torno de $1,2 \mathrm{~V}$ e outro catódico ao redor de $0,95 \mathrm{~V}$, atribuídos à formação e redução de óxidos de ouro, respectivamente. No intervalo de potencial a que corresponde os picos de redução e oxidação da espécie eletroativa $\left(\mathrm{Fe}(\mathrm{CN})_{6}{ }^{4-}\right)$ no eletrólito de suporte, não são observados picos significativos referentes à formação de óxidos de ouro. Este perfil voltamétrico do eletrodo de ouro em solução de ácido sulfúrico é indicativo de que a superfície está praticamente livre da presença de óxidos.

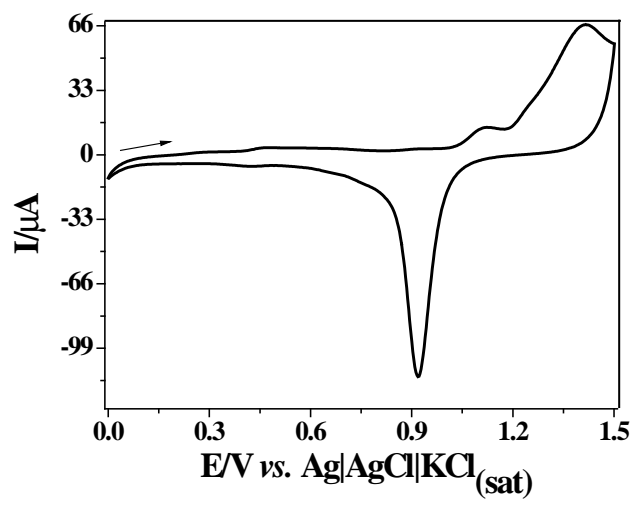

Figura 7. Voltamograma cíclico em solução de ácido sulfúrico $0,5 \mathrm{~mol} \mathrm{~L}^{-1}$ do CDtrodo de ouro; $v=100 \mathrm{mV} \mathrm{s}^{-1}$.

Após esse pré-tratamento, realizou-se as medidas voltamétricas em $\mathrm{Fe}(\mathrm{CN})_{6}{ }^{4-} 1,0 \times 10^{-3}$ mol L-1. A Figura 8-A mostra que houve mudança significativa no perfil voltamétrico do $\mathrm{Fe}(\mathrm{CN})_{6}{ }^{4-}$, $\operatorname{com} \Delta \mathrm{E}_{\text {pico }}$ de aproximadamente $77 \mathrm{mV}$ e razão de corrente de pico de 0,96. Estes valores estão bem próximos dos valores esperados para um processo de transferência eletrônica reversível que envolve um elétron, sendo assim, satisfatórios para o objetivo do estudo. O estudo de velocidade de varredura com o CDtrodo assim construído é apresentado na figura 8-B, confirmando que os processos eletródicos sobre a superfície metálica de ouro ocorrem sem complicações cinéticas.
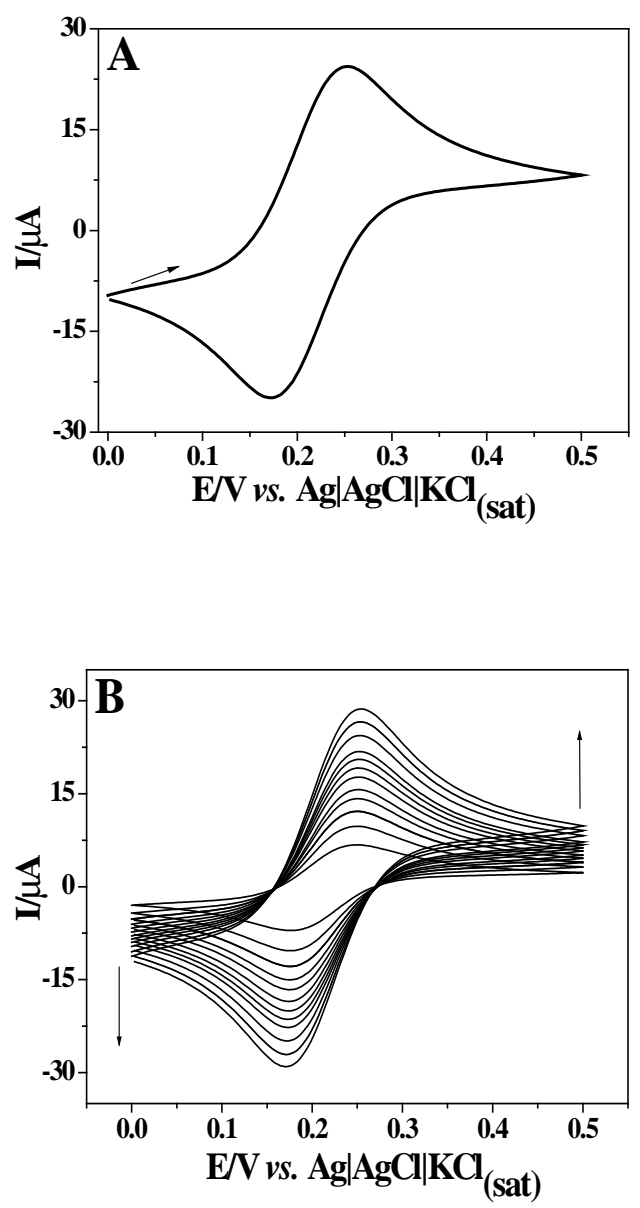

Figura 8. Voltamograma cíclico em tampão fosfato $0,1 \mathrm{~mol} \mathrm{~L}^{-1}, \mathrm{pH} \quad 7,0$ contendo $\mathrm{Fe}(\mathrm{CN})_{6}^{4-}$ $1,0 \times 10^{-3} \mathrm{~mol} \mathrm{~L}^{-1}, v=50 \mathrm{mV} \mathrm{s}^{-1}$ (A). Efeito da velocidade de varredura (B). $\mathrm{E}_{\mathrm{T}}$ : CDtrodo de ouro após limpeza por ciclagens sucessivas em $\mathrm{H}_{2} \mathrm{SO}_{4} 0,5 \mathrm{~mol}$ $\mathrm{L}^{-1}$.

O eletrodo construído com auxílio de fita de galvanoplastia e pré-tratado pelo método de limpeza de superfície com ácido sulfúrico foi então avaliado segundo sua reprodutibilidade, comparando-se os valores de picos de correntes anódica e catódica, dos processos redox relativos a espécie $\mathrm{Fe}(\mathrm{CN})_{6}{ }^{4-}$. O desvio padrão relativo medido para 5 CDtrodos diferentes, construídos e avaliados no mesmo dia, sobre as mesmas condições de trabalho, foi de 1,7\%. Este resultado indica que as áreas de trabalho dos eletrodos apresentaram-se 
muito similares, o que descreve um processo de confecção de eletrodos de CDs de ouro bastante reprodutivo.

A utilização da fita de galvanoplastia para delimitar a área geométrica do CDtrodo mostrouse viável e exequiível em qualquer laboratório porém deve se atentar para o solvente no qual serão efetuadas as medidas; em solução alcoólica a fita se desprende facilmente [17].

A investigação de diferentes tipos de CDRs de ouro disponíveis no mercado indicou que o ácido nítrico foi eficiente para a retirada da película protetora, no entanto a limpeza do CDtrodo depende da procedência do CD-R alguns podem ser empregados diretamente sem tratamento prévio, outros exigem tratamento com $\mathrm{H}_{2} \mathrm{SO}_{4}$.

\section{CONCLUSÕES}

A proposta de delimitar a área do CDtrodo empregando a fita para galvanoplastia mostrou-se viável, eficiente e de fácil construção, apresentando reprodutibilidade compatível com os eletrodos construídos a partir de CDs apresentados na literatura.
A retirada da película protetora do CD-R, empregando ácido nítrico, implica na formação de camadas de óxidos de ouro sobre a superfície metálica eletródica, requerendo então, o pré-tratamento destas superfícies, nos CDtrodos. Dentre os métodos de limpeza empregados, o melhor resultado foi obtido com ciclagem sucessiva em meio de ácido sulfúrico, até obtenção de um sinal estável compatível com os eletrodos de ouro convencionais.

O método de limpeza da superfície de ouro de um CDtrodo possui grande dependência da procedência do CD-Rs utilizados.

\section{AGRADECIMENTOS}

Os autores agradecem à Fapesp (Proc. 2007/ 04227-9) e CNPq (Proc. 150670/2007-9, 140154/2005) pelo apoio financeiro.

Evaluation of the CDtrode cleaning constructed from gold recordable $\mathrm{CD} /$ galvanoplasty tape

Received July 032008

Accepted October 142009

\begin{abstract}
Gold electrodes are widely used in electrochemistry and electroanalytical chemistry, due to the high purity, broad range of work potential as well as the possibility to control and modify the surface. On this work construction and cleaning of electrodes from gold recordables compact discs (CD-Rs), namely CDtrodes, are described. The CDs was submitted to the action of concentrated $\mathrm{HNO}_{3}$ in order to remove the protection layer and to expose the metallic layer; the electrode area was delimited by using galvanoplasty tape. The literature has been published many articles on CDtrode focused on the application but not on the cleaning; there is no register on galvanoplasty tape to define electrode area. Several methods for gold surface cleaning after attack of $\mathrm{HNO}_{3}$ were investigated, such as application of fixed potential in $\mathrm{NaCl}$ solution, successive scans in $\mathrm{H}_{2} \mathrm{SO}_{4}$ and ultrasound application, and best results were obtained in $\mathrm{H}_{2} \mathrm{SO}_{4}$ solution. This electrode material is interesting due to the performance comparable to the commercial electrodes, besides great versatility and low cost.
\end{abstract}

Keywords: CDtrode, CD-R electrodes; cyclic voltammetry; gold surface cleaning

\section{REFERÊNCIAS}

[1] J. Janata, M. Josowicz, M. Devaney, Anal. Chem. 66 (1994) 207.

[2] L. Angnes, E. M. Richter, M. A. Augelli, G. H. Kume, Anal. Chem. 72 (2000) 5503.

[3] D. Daniel, I. G. R. Gutz, Electrochem. Comm. 5 (2003) 782.

Ecl. Quím., São Paulo, 34(2): 59 - 66, 2009
[4] D. Lowinsohn, E. M. Richter, L. Angnes, M. Bertotti, Electroanalysis 18(1) (2006) 89.

[5] H-Z Yu, Anal. Chem. 73 (2001) 4743

[6] R. A. A. Muñoz, R. C. Matos, L. Angnes, J. Pharm. Sci. 90(12) (2001) 1972.

[7] E. M. Richter, M. A. Augelli, S. Magarotto, L. Angnes, Electroanal. 13(8-9) (2001) 760. 
[8] A. Walcarius, E. Sibottier, Electroanal. 17(19) (2005) 1716.

[9] E. M. Richter, J. J. Pedrotti, L. Angnes, Electroanal. 15 (2003) 2003.

[10] P. Westbroek, J. Strycker, , P. Dubruel, E. Temmerman, E. H. Schacht, Anal. Chem. 74 (2002) 915.

[11] C. F. Pereira, F. B. Gonzaga, A. M. Guaritá-Santos, J. R. Souzade, Talanta 69 (2006) 877

[12] P. C. W. Sidney, O. K. Nathan, Methods in enzimology, Academic Press Inc., New York.

[13] P. R. B. O. Marques, L. Angnes, H. Yamanaka, V Congresso Iberoamericano de Sensores Abstract Ibersensor 2006. [14] S. Campuzano, M. Pedrero, C. Montemayor, J. Electroanal. Chem. 586 (2006) 112.

[15] C. L. Perdriel, A. J. Arvia, M. Ipohorski, J. Electroanal. Chem. 215 (1986) 317.

[16] M. I. Florit, M. E. Martins, A. J. Martins, J. Electroanal. Chem. 151 (1983) 209.

[17] M. V. Foguel, Monografia - Instituto de Química, Universidade Estadual Paulista, Araraquara, 2008. 